\title{
Benefits of Sports Participation for Executive Function in Disabled Athletes
}

\author{
Francesco Di Russo,,2 Alessandro Bultrini, Stefano Brunelli,, ${ }^{3,4}$ Anna Sofia Delussu, ${ }^{3}$ \\ Lorenzo Polidori, ${ }^{4}$ Francesco Taddei, Marco Traballesi, ${ }^{3,4}$ and Donatella Spinelli, ${ }^{1,2}$
}

\begin{abstract}
We investigated the effect of sports activity on physically-disabled individuals using behavioral and electrophysiological techniques. Visual go/no-go discriminative and simple response tasks were used. Participants included 17 disabled athletes, 9 from open-skill (wheelchair basketball) and eight from closed-skill (swimming) sports, and 18 healthy non-athletes. Reaction times of the disabled athletes were slower than those of healthy non-athletes on both tasks ( $7 \%$ and 13\% difference, respectively). Intra-individual variations in reaction times, switch cost, and number of false alarms, were higher in the swimmers, but comparable to healthy non-athletes, in the basketball group. Event-related potentials (ERPs) early components P1, N1, and P2 had longer latencies in the disabled athletes. The late P3 component had longer latency and smaller amplitude in the disabled athletes only in the discriminative response task. The N2 component, which reflected inhibition/execution processing in the discriminative response task, was delayed and reduced in the swimmer group, but was comparable to healthy subjects in the basketball group. Our results show that (1) the ERP components related to perceptual processing, and late components related to executive processing, were impaired in disabled subjects; and (2) open-skill sports such as basketball may partially compensate for executive control impairment by fostering the stability of motor responses and favoring response flexibility.
\end{abstract}

Keywords: cortical plasticity; deafferentation; event-related potential; physical disability

\section{Introduction}

C URRENT RESEARCH in many areas, including physiology, biology, and biomechanics, reflects interest in the effects of sports activity on individuals with physical disabilities. Although psychological studies are few and have mostly focused on psychosocial effects, they show that participation in sports is important for improving perceived quality of life and community integration (McVeigh et al., 2009), and has benefits for those with anxiety and depression (Gioia et al., 2005). As physical activity might also have a positive effect on basic psychomotor functions, we analyzed some basic psychomotor functions and their underlying brain activity in disabled athletes.

Psychomotor functions are impaired in persons confined to a wheelchair following spinal cord injury (SCI), amputation, or neural infections, such as poliomyelitis (Plinta et al., 2005). In a large group of participants studied, simple and choice reaction times of functionally-intact upper limbs to visual stimuli were slower than those of healthy, able-bodied controls. Research on sensory deprivation suggests that loss of somatosensory input to the central nervous system may have an impact on cortical reactivity and subsequent cognitive task efficiency in individuals with SCI (Crossman, 1996). Few electrophysiological data show that cognitive brain responses to tactile and auditory tasks are attenuated in SCI subjects (Ament et al., 1995; Choen et al., 1996); moreover, P3 components of visual stimuli have longer latencies than normal in amputees (Karl et al., 2004).

Studies in healthy athletes show that extensive daily physical training modifies cortical activity (Hatfield and Hillman, 2001; Nakata et al., 2010). Increased neural efficiency (i.e., performing the same work with less cortical effort) has been demonstrated in athletes using electrophysiological techniques. However, the reverse effect has been reported for sensory stimuli processing (Murakami et al., 2008; Özmerdivenli et al. 2005), and when attention and motor inhibition are required (Di Russo et al., 2006). These data can be interpreted

\footnotetext{
${ }^{1}$ Department of Education Sciences for Motor Activity and Sport, University of Rome "Foro Italico," Rome, Italy.

${ }^{2}$ Laboratory of Neuropsychology, Santa Lucia Foundation (IRCCS), Rome, Italy.

${ }^{3}$ Santa Lucia Foundation (IRCCS), Rome, Italy.

${ }^{4}$ Santa Lucia Sport, Rome, Italy.
} 
as adaptive changes consequent to sports activity. In agreement with the neural efficiency theory, motor preparation requires less cortical activity (Di Russo et al., 2005a, 2005b); however, enhancement of sensory and cognitive event-related potential (ERP) components is consistent with top-down attentional modulation of input and output associated with excellent performance on specific tasks. Although the effects of sports on the brain are well documented in healthy athletes, no data are available on disabled athletes.

As different sports activities require different sets of skills, we selected two sports that require very different cognitive and executive process skills, namely basketball and swimming (executive processes include planning, cognitive flexibility, abstract thinking, rule acquisition, initiating appropriate actions and inhibiting inappropriate actions, as well as selecting relevant sensory information). Sports such as basketball, fencing, or football usually involve "openskills," because the environment constantly changes and movements have to be continually adapted. These skills are predominantly perceptual and externally-paced (e.g., a pass in basketball). By contrast, "closed-skill" sports, like swimming, archery, and golf, take place in a stable, predictable environment, and the performer knows exactly what to do and when to do it. Therefore, the skills needed are not affected by the environment, and tend to be habitual. Movements follow set patterns and have a clear beginning and end; these skills tend to be self-paced.

We used a go/no-go task that required typical open-skill sports skills. This task mimics some of the complexity of the visual motor behavior of basketball players. These players have to respond to the opponent's actions, and often have to switch from an intended action to a new one that is more appropriate for the changing situation. In the go/no-go task, the subject has to produce a fast motor response to target stimuli, and to refrain from responding to other non-target stimuli. This paradigm involves many perceptual, cognitive, and motor processing stages: (1) task preparation, during which the relevant task set is activated (in these experiments this was called "prepare to discriminate among several stimuli"); (2) stimulus perception and identification; (3) stimulus response mapping (i.e., selecting a stimulus category and mapping it to the assigned response); (4) response execution or response inhibition; and (5) response monitoring (error versus correct response) and adjustment of attention. Besides their relevance in open-skill sports, these processes underlie the ability to cope quickly and appropriately with the changing environment in everyday life (e.g., when driving, switching from the accelerator to the brakes at a red light).

Different experimental conditions (i.e., discrimination difficulty, multiple motor response choices, and the occurrence of go and no-go trials) modulate the processing load in the various stages of the go/no-go task. In accordance with the literature, to focus on motor execution and inhibition (rather than interference, conflict, or error processing), we used an equal probability of go and no-go stimuli. The task required relatively simple feature discrimination (targets versus nontargets); moreover, single motor responses had to be made (go trials) or withheld (no-go trials). In another experiment we used a simple reaction task in which stimulus discrimination was not required, and the participants had to press a key at the appearance of all visual stimuli.
Relevant information on the neural mechanisms underlying performance can be obtained by simultaneously recording behavior and brain activity. In the go/no-go task, various ERP components are recorded. The early components (P1, N1, and $\mathrm{P} 2)$, which are typically present during any visual task, are followed by two later components specifically modulated by the go/no-go condition: first a negative component (N2), and then a positive deflection (P3). The N2 component is maximal in fronto-central sites, and is larger in the no-go than in the go condition. N2 peaks around $250-300 \mathrm{msec}$, is maximal at medial frontal sites, and is considered an important marker of motor inhibitory processes (Di Russo et al., 2006; Di Russo and Spinelli, 2009). The P3 component (latency range 300$600 \mathrm{msec}$ ) is maximal in the centro-parietal site for go trials, and in the fronto-central sites for no-go trials (Di Russo and Spinelli, 2009; Di Russo et al., 2006; Salisbury et al., 2004), and reflects multiple processing events related to the stimulus and response evaluation.

Only one study of basketball paralympic athletes has evaluated the contributions of selected fundamental factors to performance as measured by season statistics and coach evaluations. It showed that choice reaction tasks (RTs), not simple RTs, contribute to performance (Wang et al., 2005). Thus basketball players should have an advantage over swimmers only in the discrimination task. However, the ability to react quickly to a stimulus when executing the same response (simple RT) is required in both sports. Thus, at the behavioral level, sports should improve this simple reaction time skill in both disabled groups, and compensate for the impairment seen compared to able-bodied controls, as found by Plinta and associates (2005).

Note that electrophysiological components are particularly useful for detecting differences between groups when overt responses are unavailable (i.e., in the no-go condition). The ability to inhibit a programmed action is common in basketball players, and is reflected mainly as an enhanced N2 component.

\section{Methods \\ Subjects}

The study included 35 participants: 17 disabled athletes, including nine wheelchair basketball players (mean age 35.3 years; SD 1.9 years), and eight swimmers (mean age 33.3 years; SD 9.9 years), and 18 healthy non-athletes (mean age 34.8 years; SD 4.6 years). The three groups were matched for age, gender, education level, and hand preference. Both the basketball players and the swimmers suffered from physical disability of the lower limbs (Table 1). Most of the patients suffered SCI and amputations, which in all cases were due to accidents. The presence of severe traumatic brain injury (TBI) followed by coma was recorded in only one case (DA \#1); however, this case showed no cognitive impairment. Time after onset was very long, with an average of 16.1 years (range 6-34 years). In two disabled athletes, the etiology was poliomyelitis infection in early infancy.

The athletes had at least 6 years of sports experience, and had participated in national and international championships; some also played on the Italian Paralympics team. On average, they had 9.5 years of experience, and practiced their sport 8-12 h per week. All disabled athletes except two (one in the basketball group and one in the swimmer group) did not 
Table 1. Demographic and Clinical Data of the Disabled Athletes

\begin{tabular}{lclll}
\hline & Age $(y)$ & Sex & Sport & \multicolumn{1}{c}{ Etiology } \\
\hline DA 1 & 35 & M & Basketball & SCI \\
DA 2 & 31 & M & Basketball & SCI \\
DA 3 & 35 & M & Basketball & SCI \\
DA 4 & 40 & M & Basketball & SCI \\
DA 5 & 37 & M & Basketball & BKA \\
DA 6 & 34 & M & Basketball & BKA \\
DA 7 & 36 & M & Basketball & AKA \\
DA 8 & 36 & M & Basketball & Poliomyelitis \\
DA 9 & 34 & M & Basketball & Poliomyelitis \\
DA 10 & 39 & M & Swimming & SCI \\
DA 11 & 50 & M & Swimming & SCI \\
DA 12 & 38 & M & Swimming & SCI \\
DA 13 & 26 & M & Swimming & SCI \\
DA 14 & 23 & F & Swimming & SCI \\
DA 15 & 38 & M & Swimming & BKA \\
DA 16 & 28 & M & Swimming & BKA \\
DA 17 & 24 & M & Swimming & AKA \\
\hline
\end{tabular}

DA, disabled athlete; SCI, spinal cord injury; BKA, below knee amputation; AKA, above knee amputation; SCI and amputation were the result of accidents.

participate in competitive sports before their disability. Healthy participants were mostly sedentary; some took part in sports activities on a non-professional level for an average of $1.1 \mathrm{~h}$ per week. All subjects provided written informed consent to participate in the experiment after the procedures (which were approved by the local ethics committee) had been fully explained to them.

\section{Stimuli}

The fixation point was a $\operatorname{dot}\left(0.3^{\circ} \times 0.3^{\circ}\right.$ of the visual field $)$ in the center of a computer screen. Four configurations composed of vertical and horizontal light grey bars subtending $4^{\circ} \times 4^{\circ}$ were presented for $260 \mathrm{msec}$ on a dark grey background. The lower edge of the stimulus was centered $1^{\circ}$ above the fixation point to stimulate only the upper hemifield, and to avoid concurrent activity in visual areas with opposite geometry (such as V1), that might lead to the reduction of some ERP components. The four configurations were displayed randomly with equal probability $(p=0.25)$; stimulus onset asynchrony varied from 1-2 sec.

\section{Procedure}

In separate runs, the subjects performed two tasks: a discriminative reaction task (DRT) and a simple reaction task (SRT). In the DRT, two configurations were defined as targets and two as non-targets. The subjects had to press a key with their right hand as quickly as possible when a target appeared on the screen (go stimuli; $p=0.5$ ) and had to refrain from responding when a non-target appeared (no-go stimuli; $p=0.5$ ). The types go and no-go stimuli were counterbalanced across subjects. In the SRT, the subjects had to respond to all four configurations. Five runs of the SRT and 10 runs of the DRT consisted of a sequence of 400 SRT, go, and no-go trials each. The order of the tasks was evenly distributed. Only trials followed by a correct response in the 100-1000 msec window were counted (responses below $100 \mathrm{msec}$ were considered stimulus anticipations, and responses above $1000 \mathrm{msec}$ were rejected because of the possible overlap with the presentation of the following stimulus). We excluded the first trial of each run from the analysis to avoid orienting response contamination and to provide warm-up trials. The order of presentation was randomized across subjects. The duration of each run was $2 \mathrm{~min}$, followed by a pause (total duration = about $45 \mathrm{~min})$.

\section{Analysis of the reaction tasks}

To investigate the RTs, variability between groups, the intra-individual variability of RTs was obtained using the intra-individual coefficient of variation (ICV): ICV = standard deviation of RT/mean of RT, and was calculated for each subject for each task, including all responses above $100 \mathrm{msec}$. The ICV measures performance stability controlled to some degree for speed of response (Stuss et al., 2003). To quantify the flexibility of motor responses (i.e., the cost of switching from inhibition to action), the switch cost was calculated in the DRT condition, as the difference between RT following a no-go trial (switch condition), and RT following a go trial (non-switch condition).

Median RTs, ICV, and omissions were analyzed using a $3 \times 2$ analysis of variance (ANOVA), including the group (basketball players versus swimmers versus healthy nonathletes), and task (SRT versus DRT) as factors. Switch costs and false alarms were analyzed using a one-way ANOVA comparing the three groups. Post-hoc comparisons were conducted using the Tukey honest significant difference (HSD) test. The significance level was set at $p=0.05$. To estimate the size of the statistical effects, the partial eta squared $\left(\eta_{\mathrm{p}}{ }^{2}\right)$ was calculated, in addition to probability values.

To evaluate the general effect of sports activity on the disabled, we compared the data from the disabled athletes in our study with previous data reported by Plinta and colleagues (2005). The comparison was possible for the SRT, because the two tasks were very similar, but not for the DRT, because the tasks were too dissimilar. Moreover, the comparison was possible only for the SCI subjects (the amputee and neural infection samples were too small in our study to allow comparison). We selected 12 SCI participants from the sample of 17 disabled athletes, and compared their performance data with the SCI data from the other study $(n=48$; group A, Table 1 in Plinta et al., 2005). For both studies, we normalized the performance of SCI subjects with respect to those of healthy non-athletes using the formula: ((RT of SCI - RT of healthy non-athletes)/RT of healthy nonathletes) $\times 100$.

\section{Electrophysiological recording and analysis}

Electrophysiological recording was carried out while the participants performed the tasks. Electroencephalography (EEG) was recorded using the BrainVision ${ }^{\text {TM }}$ system (Brain Products $\mathrm{GmbH}$, Munich, Germany), with 64 sensors referenced to the left mastoid. Horizontal eye movements, blinks, and vertical eye movements were recorded. The EEG was digitized at $250 \mathrm{~Hz}$, amplified (band-pass of $0.01-60 \mathrm{~Hz}$, including a 50-Hz notch filter), and stored for off-line averaging. Prior to signal averaging, the data were re-referenced to the linked mastoids, and artifact rejection was performed to discard contaminated epochs $(13 \%$ of the trials were rejected, 
mostly for eye blinks). ERPs were averaged in epochs starting $100 \mathrm{msec}$ prior to stimulus onset, and lasting for $1100 \mathrm{msec}$. To further reduce high- and low-frequency noise, the timeaveraged ERPs were band-pass filtered from 0.05 to $25 \mathrm{~Hz}$. To visualize the voltage topography of the ERP components, spline-interpolated 3D maps were constructed using BESA 2000 software (MEGIS software GmbH, Gräfelfing, Germany).

ERPs from the SRT and DRT runs were sorted into three categories: (1) ERPs for SRT stimuli, (2) ERPs for DRT go stimuli, and (3) ERPs for DRT no-go stimuli. Peak amplitudes (measured with respect to the $100 \mathrm{msec}$ pre-stimulus baseline) and latencies of the major ERP components were calculated for each subject in the following time windows: P1 (80$150 \mathrm{msec}), \mathrm{N} 1$ (130-200 msec), P2 (180-300 msec), N2 (200$350 \mathrm{msec})$, and P3 (250-600 msec). The component identification was also guided by their polarity and topography, as previously described (Di Russo and Spinelli, 2009; Di Russo et al., 2006; Salisbury et al., 2004).

\section{Statistical analysis}

Data from the P1, N1, and P3 components were evaluated with a $3 \times 3$ ANOVA, including group (basketball players versus swimmers versus healthy non-athletes), and task (SRT, go, and no-go). For the N2, a $3 \times 2$ ANOVA was used, because this component was only present in the go and no-go conditions. As P2 was present only in the SRT condition, it was analyzed using one-way ANOVA (Table 2). To estimate the size of the statistical effects, the partial eta squared $\left(\eta_{\mathrm{p}}{ }^{2}\right)$ was also calculated. Post-hoc comparisons were conducted using the Tukey HSD test. The overall alpha level was fixed at 0.05 .

\section{Results}

\section{Behavioral data}

Simple reaction task (SRT). Accuracy on the SRT was high and comparable in the three groups (Fig. 1a), and the percentages of rejected trials were $3.4 \%, 4.7 \%$, and $1.7 \%$, in basketball players, swimmers, and healthy non-athletes, respectively. Most rejections (more than $97.6 \%$ ) were stimulus anticipations. Mean simple reaction times and ICV on the SRT are reported in Figure $1 \mathrm{~b}$ and c, respectively. Post-hoc analysis showed that healthy non-athletes were about $30 \mathrm{msec}$ faster $(p<0.001)$ than disabled athletes; the difference between the two groups of disabled athletes was not significant. ICV was higher in swimmers $(p<0.05)$ than in the other two groups, which did not differ significantly (Fig. 1c).

To evaluate the general effect of sports activity on SCI subjects (as illustrated in the methods section), we compared the disabled athletes' data with the data reported by Plinta and associates (2005). The slowing down of simple reaction times of SCI subjects was $14 \%$ in the sedentary group (Plinta et al., 2005) versus $7 \%$ in the athletes (in the present study).

Discriminative reaction task (DRT). In the DRT, the number of false alarms (Fig. 1d) showed a significant group effect $\left(\mathrm{F}_{2,34}=5.14, p<0.01, \eta_{\mathrm{p}}{ }^{2}=0.257\right)$, indicating that swimmers made more false alarms (post-hoc: $p<0.01$ ) than the other two groups, which had similar results.

ANOVA on DRT reaction times showed a significant group effect $\left(\mathrm{F}_{2,34}=5.89, p<0.01, \eta_{\mathrm{p}}{ }^{2}=0.365\right)$. Post-hoc analysis showed that healthy non-athletes were $57 \mathrm{msec}(11.5 \%)$ faster $(p<0.001)$ than disabled athletes; the difference between the two groups of disabled athletes was not significant (Fig. 1e). Rejected trial percentages were $1.8 \%, 3.4 \%$, and $0.8 \%$, in basketball players, swimmers, and healthy non-athletes, respectively. Stimulus anticipations accounted for more than $96.2 \%$ of the rejections.

The ANOVA group effect on ICV was also significant $\left(\mathrm{F}_{2,34}=9.79, p<0.001, \eta_{\mathrm{p}}{ }^{2}=0.257\right)$; post-hoc analysis showed that ICV was higher in swimmers $(p<0.05)$ than in the other two groups, which did not differ significantly from each other (Fig. 1f).

The switch cost on DRT (Fig. 1g) showed a significant group effect $\left(\mathrm{F}_{2,34}=4.18, p<0.05, \eta_{\mathrm{p}}{ }^{2}=0.198\right)$. Post-hoc com-

Table 2. Analysis of Variance Results for Event-Related Potential (ERP) Components

\begin{tabular}{|c|c|c|c|c|c|c|c|c|}
\hline & Effect DF & Error DF & F value & p Value & $\eta_{p}{ }^{2}$ & F value & p Value & $\eta_{p}{ }^{2}$ \\
\hline \multicolumn{9}{|c|}{ ERP component } \\
\hline $\mathrm{P} 1$ & & & Latency & & & Amplitude & & \\
\hline Group & 2 & 34 & 3.70 & $<0.05$ & 0.149 & $<1$ & ns & 0.023 \\
\hline Task & 2 & 34 & 1.51 & ns & 0.035 & $<1$ & ns & 0.021 \\
\hline Group $\times$ task & 4 & 68 & $<1$ & ns & 0.028 & $<1$ & ns & 0.026 \\
\hline N1 & & & Latency & & & Amplitude & & \\
\hline Group & 2 & 34 & 6.01 & $<0.01$ & 0.261 & $1.02^{1}$ & ns & 0.095 \\
\hline Task & 2 & 34 & $<1$ & ns & 0.025 & 4.75 & $<0.05$ & 0.135 \\
\hline Group $\times$ task & 4 & 68 & $<1$ & ns & 0.049 & 1.75 & ns & 0.106 \\
\hline $\mathrm{P} 2$ & & & Latency & & & Amplitude & & \\
\hline Group & 2 & 34 & 4.22 & $<0.05$ & 0.156 & 1.63 & ns & 0.068 \\
\hline N2 & & & Latency & & & Amplitude & & \\
\hline Group & 2 & 34 & 15.22 & $<0.01$ & 0.420 & 3.23 & $<0.05$ & 0.133 \\
\hline Task & 1 & 34 & 1.81 & ns & 0.041 & 10.22 & $<0.01$ & 0.196 \\
\hline Group $\times$ task & 2 & 34 & $<1$ & ns & 0.012 & 2.81 & ns & 0.099 \\
\hline P3 & & & Latency & & & Amplitude & & \\
\hline Group & 2 & 34 & 4.44 & $<0.05$ & 0.175 & 4.17 & $<0.05$ & 0.147 \\
\hline Task & 1 & 34 & 123.89 & $<0.01$ & 0.746 & 11.54 & $<0.01$ & 0.456 \\
\hline Group $\times$ task & 4 & 68 & 3.58 & $<0.05$ & 0.110 & 3.45 & $<0.05$ & 0.105 \\
\hline
\end{tabular}

ns, not significant; DF, degrees of freedom. 

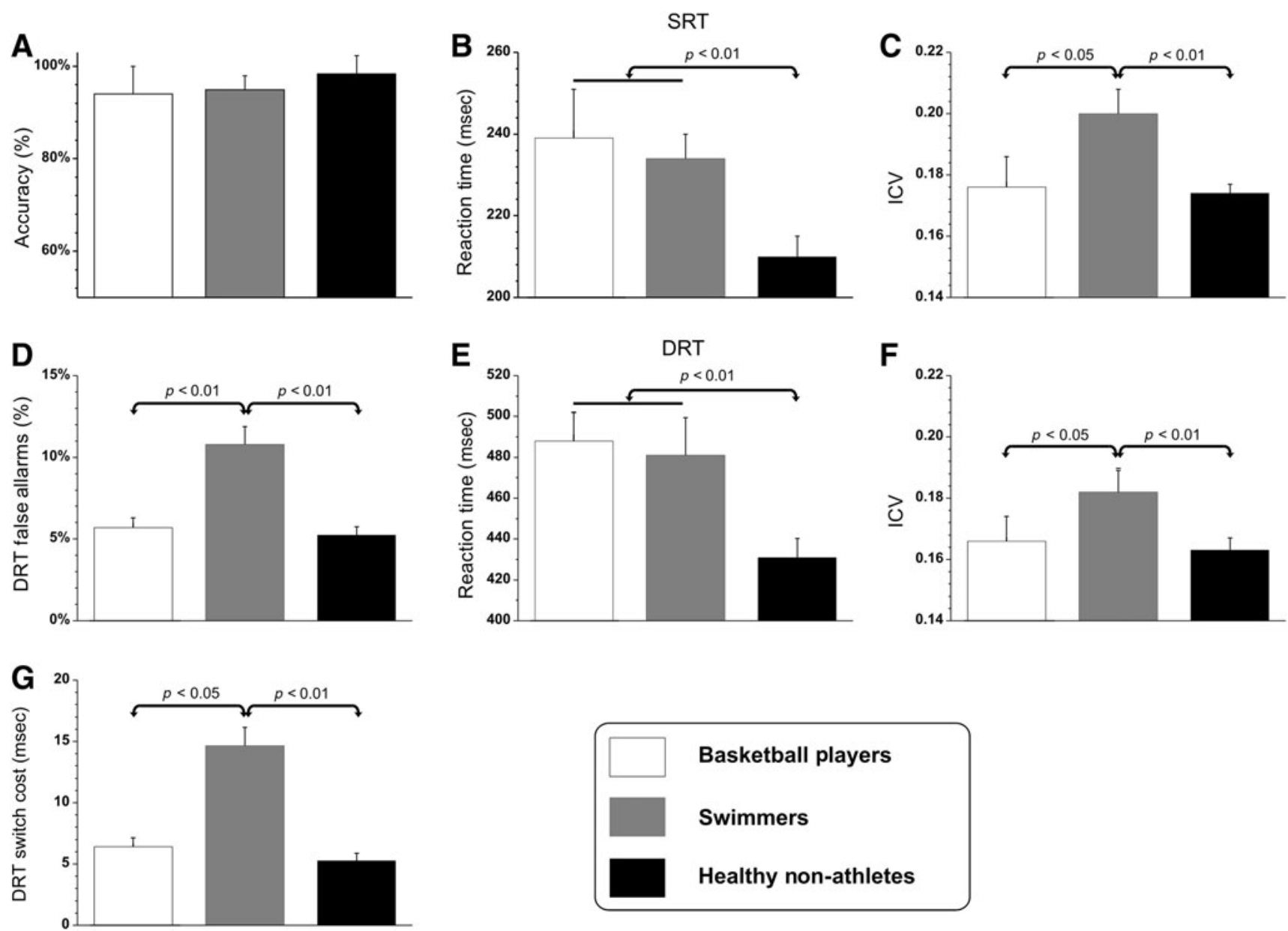

FIG. 1. Behavioral measures: means and standard deviations of the three groups. Upper panels, simple reaction task (SRT): (A) accuracy, and (B) reaction time to visual stimuli. (C) Intra-individual coefficient of variation (ICV) of the reaction time. Middle and lower panels, discriminative reaction task (DRT): (D) false alarms for no-go trials, (E) mean DRT, and (F) ICV. (G) Switch cost for switching from no-go to go trials.

parisons indicated that swimmers' switch cost was larger $(p<0.01)$ than that of the other two groups, which did not differ from each other.

Table 3 shows the individual behavioral data of all disabled athletes.

\section{Electrophysiological data}

Main ERP waveforms and voltage topographies are shown in Figures 2 and 3, respectively. Results of the statistical analyses are shown in Table 2.

The earliest component (P1) peaked at approximately $115 \mathrm{msec}$ at bilateral parieto-occipital sites. The P1 amplitudes did not differ between groups or tasks. Analysis of P1 latency showed a significant group effect. Post-hoc analysis showed that in all conditions (SRT, go, and no-go), healthy nonathletes had a shorter P1 latency than athletes $(p<0.05)$, who did not differ significantly from each other (Fig. 4a). The difference in latency was approximately $12 \mathrm{msec}$.

At approximately $180 \mathrm{msec}$, a second component, N1, peaked at bilateral occipital sites. The N1 amplitudes did not differ between groups. However, as for P1, the N1 latency was more delayed in disabled athletes (post-hoc: $p<0.05)$ than in healthy non-athletes (Fig. 4b). The difference in latency was approximately $17 \mathrm{msec}$.

The P2 component, which was evident only in the SRT condition, peaked at approximately $250 \mathrm{msec}$ in bilateral parietal-occipital sites, and was delayed in disabled athletes (post-hoc: $p<0.05$ ). The mean size of the group difference was $20 \mathrm{msec}$ (Fig. 4c). P2 amplitudes did not differ significantly among groups.

The N2 component peaked around $280 \mathrm{msec}$ at medial central sites in the go condition, and shifted anteriorly in the no-go condition (Figs. 2 and 3b). Statistical analysis shows that the swimmers' N2 latency was about $45 \mathrm{msec}$ longer than that of the other two groups (post-hoc: $p<0.05$ ). Basketball players and healthy non-athletes did not statistically significantly differ (Fig. 5a). Also, the swimmers' N2 amplitude was lower (post-hoc: $p<0.05$ ) than that of the other two groups, which did not differ significantly from each other (Fig. 5b). A significant task effect on amplitude was present (Table 2), indicating that the N2 amplitude was larger in no-go than in go trials.

Finally, the P3 component peaked at about $350 \mathrm{msec}$ at the medial parietal sites in the SRT condition (Fig. 3b), showing similar latency and amplitude in all groups (Fig. 6). In the 
Table 3. Individual Data for the Disabled Athletes in Simple (SRT) and Discriminative (DRT) Reaction Tasks

\begin{tabular}{|c|c|c|c|c|c|c|c|c|}
\hline & \multicolumn{3}{|c|}{$S R T$} & \multicolumn{5}{|c|}{$D R T$} \\
\hline & $R T$ (msec) & $I C V$ & Accuracy & $R T$ (msec) & $I C V$ & Accuracy & False alarms & Switch cost (msec) \\
\hline DA 1 & 238 & 0.283 & 99.5 & 517 & 0.143 & 100.0 & 4.8 & 0.9 \\
\hline DA 2 & 229 & 0.191 & 96.5 & 514 & 0.175 & 99.5 & 5.3 & 15.6 \\
\hline DA 3 & 242 & 0.225 & 97.3 & 513 & 0.162 & 94.8 & 3.5 & 8.4 \\
\hline DA 4 & 215 & 0.191 & 90.8 & 478 & 0.198 & 93.5 & 10.5 & 9.8 \\
\hline DA 5 & 245 & 0.173 & 99.3 & 411 & 0.164 & 100.0 & 4.0 & 16.9 \\
\hline DA 6 & 242 & 0.182 & 97.5 & 509 & 0.179 & 99.0 & 7.5 & 6.6 \\
\hline DA 7 & 262 & 0.185 & 98.3 & 550 & 0.184 & 97.8 & 4.5 & 2.1 \\
\hline DA 8 & 222 & 0.174 & 92.3 & 470 & 0.169 & 100.0 & 10.0 & 6.0 \\
\hline DA 9 & 260 & 0.283 & 97.8 & 446 & 0.122 & 99.3 & 1.3 & 8.4 \\
\hline DA 10 & 212 & 0.191 & 93.8 & 450 & 0.221 & 97.8 & 20.1 & 14.1 \\
\hline DA 11 & 235 & 0.225 & 93.5 & 538 & 0.172 & 93.8 & 4.7 & 4.3 \\
\hline DA 12 & 293 & 0.191 & 98.8 & 554 & 0.140 & 97.3 & 11.5 & 24.1 \\
\hline DA 13 & 214 & 0.173 & 95.8 & 445 & 0.184 & 93.3 & 7.2 & 25.5 \\
\hline DA 14 & 219 & 0.185 & 94.8 & 439 & 0.165 & 97.5 & 5.0 & 29.9 \\
\hline DA 15 & 208 & 0.182 & 97.8 & 447 & 0.198 & 99.0 & 17.7 & 0.4 \\
\hline DA 16 & 265 & 0.283 & 90.8 & 452 & 0.203 & 98.0 & 17.5 & 9.8 \\
\hline DA 17 & 227 & 0.174 & 97.3 & 525 & 0.171 & 96.5 & 3.2 & 9.2 \\
\hline
\end{tabular}

Accuracy and false alarms are expressed as percentages. For ICV and switch cost computations, see methods section.

DA, disabled athlete; RT, median reaction time; ICV, intra-individual coefficient of variation of the reaction time.

DRT condition, P3 latency was longer than in the SRT (i.e., peaking at about $450-500 \mathrm{msec}$ ) and, as for the N2, its distribution shifted anteriorly (i.e., at about $450-500 \mathrm{msec}$ ) from central to frontal sites (Fig. 3b). ANOVA on P3 latency showed a significant group $\times$ task interaction (Table 2). Posthoc comparisons indicated that the go and no-go P3 latency of healthy non-athletes was earlier $(p<0.05)$ than that of the disabled athletes, who did not differ significantly from each other. The difference between disabled athletes and healthy non-athletes was $52 \mathrm{msec}$ in the go condition, and $107 \mathrm{msec}$ in the no-go condition (Fig. 6a). The P3 amplitude was reduced $(p<0.05)$ in both groups of disabled athletes (Fig. 6b). The mean size of the group difference (difference between groups) was $2.2 \mu \mathrm{V}$.

\section{Discussion}

The results of this study confirm that lower-limb-impaired subjects have longer upper-limb motor reaction times to visual stimuli than healthy non-athletes. They also extend our knowledge by demonstrating that ERP components related to perceptual processing, and late components related to executive processing, are both delayed. The consistency of findings across the different measurements suggests strong internal reliability of the results. This study also shows that an open-skill sport may partially compensate for executive control impairment, by fostering stability of motor responses and favoring response flexibility.

At the behavioral level, the RT difference between disabled athletes and healthy non-athletes was present in the simple reaction task (SRT) condition, in which the disabled athletes had slower response times. When we compared the SCI data of the athletes in this study with normative data from sedentary SCI patients (Plinta et al., 2005), we found that the athletes' delay (7\%) was half that reported in the earlier study. We interpret this reduced effect as a general advantage of sports participation over a sedentary lifestyle. In the dis- criminative reaction task (DRT), the RT difference between SCI athletes and healthy non-athletes was higher than in the SRT task $(11.5 \%)$. We cannot compare this result with normative data, because the tasks performed in the study by Plinta and associates (2005) differed from ours (i.e., they involved multiple choice, not motor inhibition).

In support of the notion that open-skill sports may improve executive control, the ICVs of basketball disabled athletes and healthy non-athletes were comparable; by contrast, swimmers had larger inter-trial fluctuations. Moreover, on the DRT, basketball disabled athletes' accuracy and switch costs were comparable to those of healthy non-athletes, whereas the swimmers' switch costs were larger. Thus basketball, but not swimming, may help to compensate for inconsistency in repeated assessment of visual-motor responses (as indicated by the ICV), and for reduced flexibility in switching from inhibition to action (as indicated by switch costs). In swimmers, difficulty in inhibiting action was also shown by their larger number of false alarms compared to the other groups.

The slowing of early electrophysiological visual components in the disabled participants is a novel result of these experiments. The P1, N1, and P2 components mainly represent stimulus-related activity in the occipital areas, and are also sensitive to selective attention (Di Russo et al., 2003, 2005b; Martinez et al., 2006). The delay in these components with respect to healthy non-athletes ranged from $12-20 \mathrm{msec}$, which is a sizable difference; note that a similar trend has been reported as an effect of age in old subjects versus young subjects (Curran et al., 2001). The slowing of processing and transmission across cortical areas was not associated with amplitude reductions or loss of stimulus information. Although RTs were slower in the disabled participants, accuracy was similar in the three groups, except for the swimmers' rates of false alarms. However, false alarms do not represent a loss of information, but rather indicate a specific problem with response inhibition. Overall, the slowing of visual processing, as indicated by P1, N1, and P2, contributed (by $\sim 20 \mathrm{msec}$ ) to 


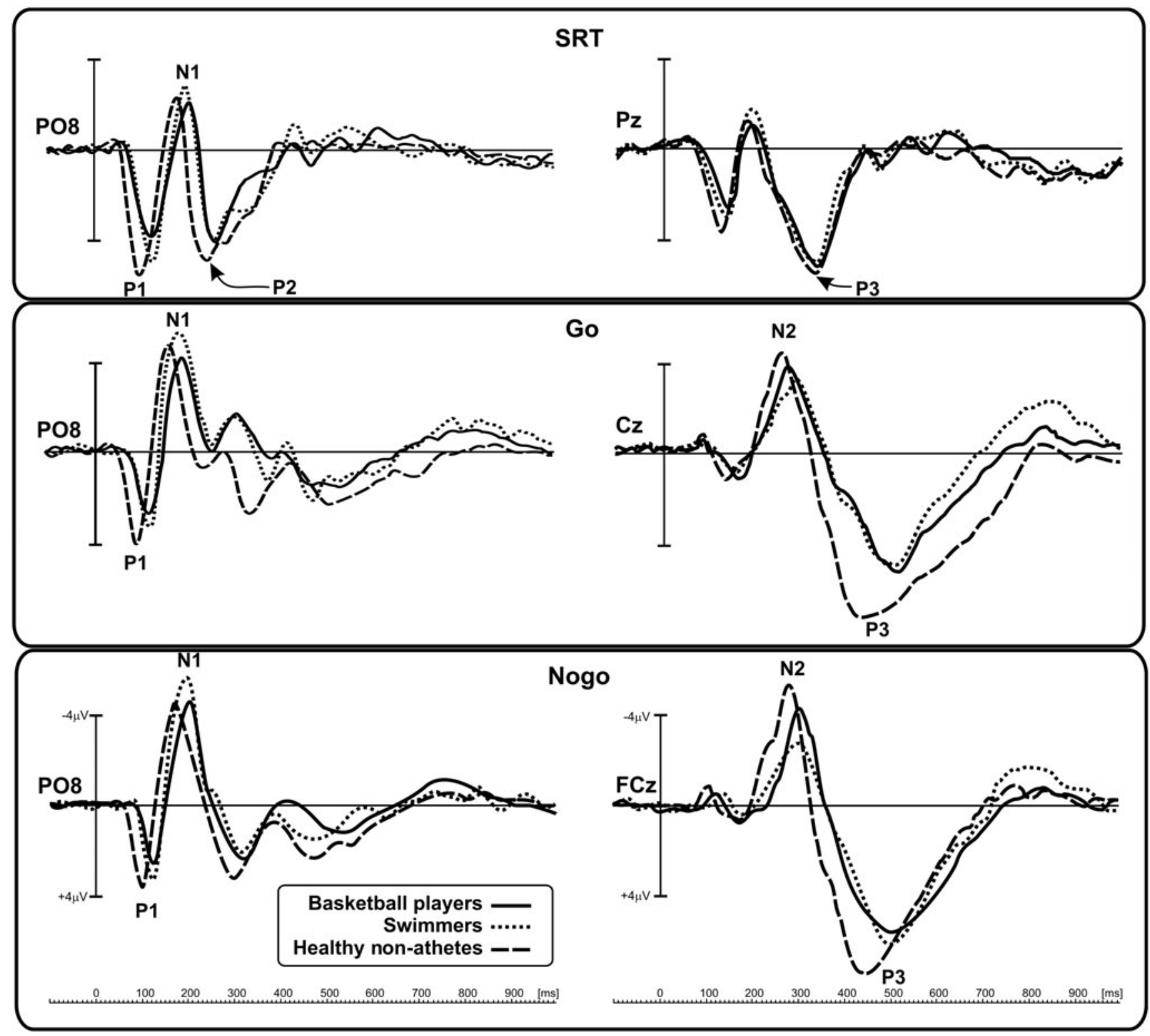

FIG. 2. Event-related potential (ERP) time course in the three groups for the studied tasks. Top panel, simple reaction task (SRT); middle and bottom panels, discriminative reaction task (DRT). Left side: Early visual processing marked by the P1, N1, and P2 components, recorded at the representative right posterior parieto-occipital sensors (PO8). Right side: Late cognitive processing associated with the N2 and P3 components, recorded at medial-parietal (Pz), central (Cz), and fronto-central (FCz) sensors.

the marked (on average $60 \mathrm{msec}$ ) RT slowing observed in both disabled groups.

The late task-related components (N2 and P3) reflect cognitive-executive processing in the parietal and frontal areas. In agreement with other reports, the N2 component was larger in the no-go than the go trials. Moreover, a clear "anteriorization" (Fallgatter and Strik, 1999) toward frontal leads was detected in the no-go condition versus the go condition, for which $\mathrm{N} 2$ peaked at medial central sites (Fig. 3b). Overall, in these experiments, the N2 component was strongly related to action inhibition/execution processing.

Reduced N2 amplitudes have been reported in various studies (e.g., in children with attention-deficit/hyperactivity disorder; Pliszka et al., 2000). These data were interpreted as difficulty inhibiting overbearing behavior. The reduced N2 amplitude (and longer latencies) seen here in disabled swimmers with respect to healthy non-athletes on the DRT might be due to executive level impairment. The reduced control of execution in swimmers is expressed at the behavioral level by a higher number of false alarms (inhibition impairment), larger ICV (instability of RTs), and higher switch costs (longer RTs when shifting from inhibition to action). By contrast, the open-skill sport enhances executive control by compensating for the disability factor; basketball athletes' N2 was comparable in amplitude and latency to those of healthy non-athletes. Furthermore, the behavioral data on false alarms, ICV, and switch costs matched the data of healthy non-athletes. 


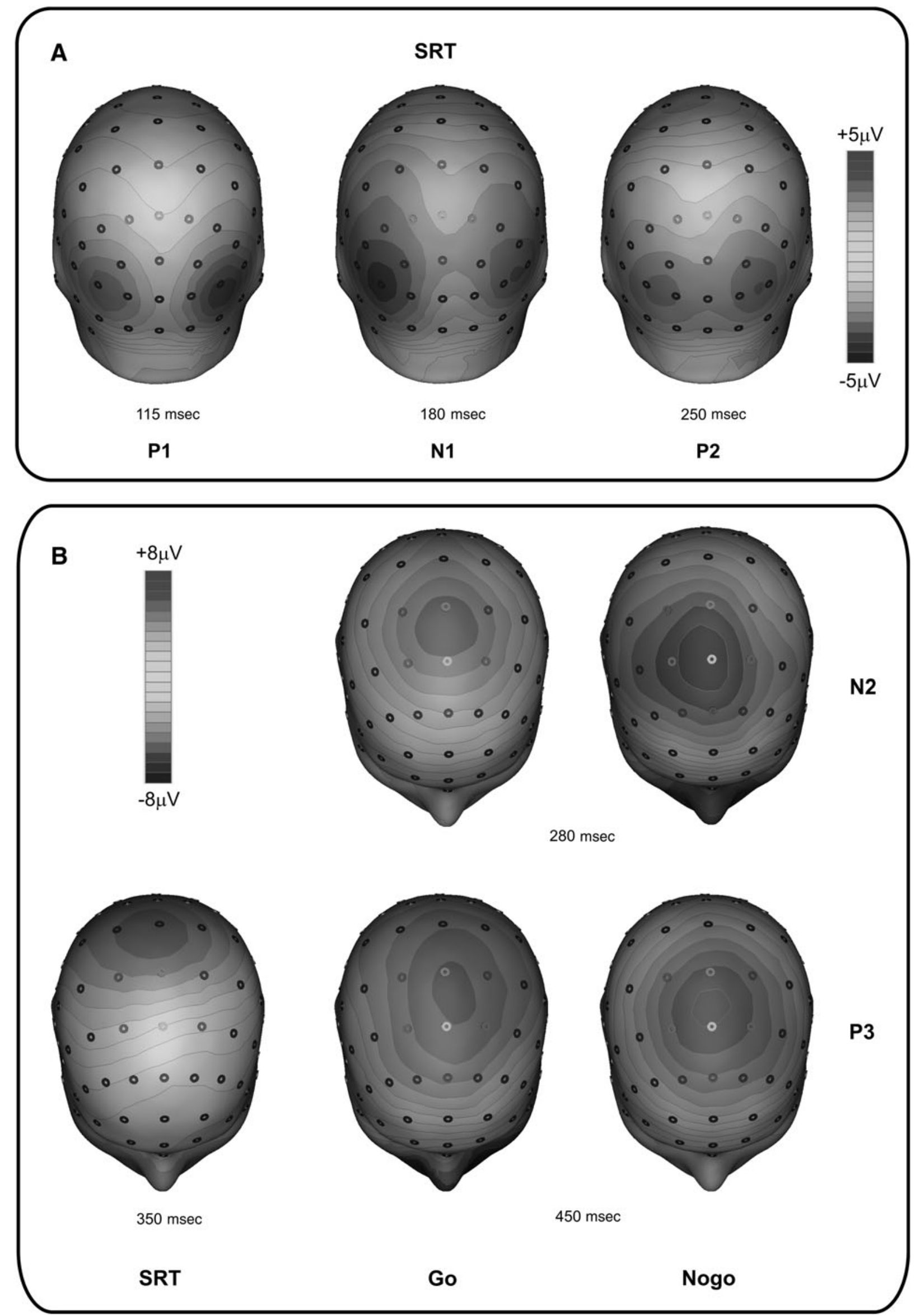



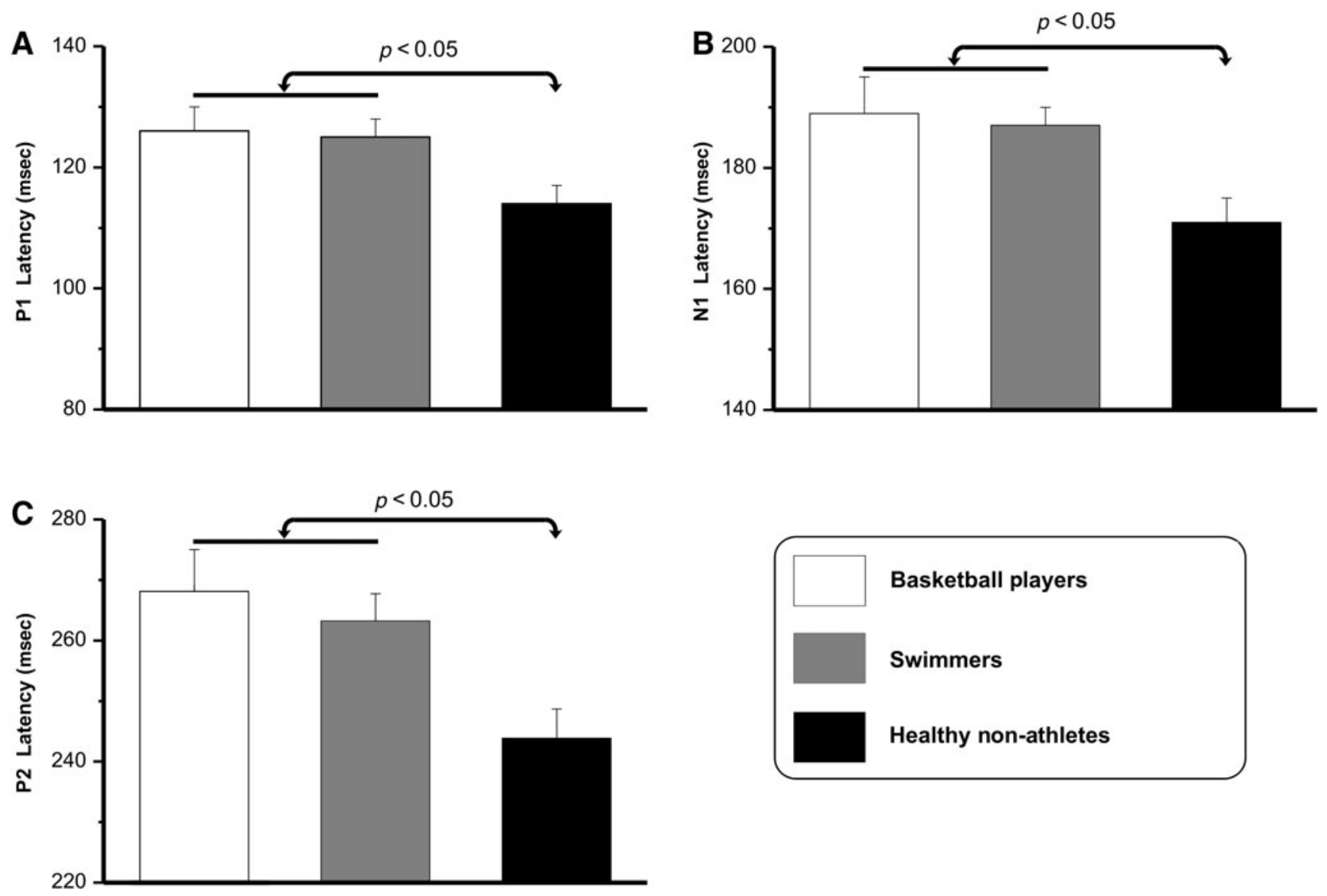

FIG. 4. Event-related potential (ERP) early components, showing significant differences between the groups: Means and standard deviations of the three groups. (A) P1 component latency averaged across the simple reaction task (SRT) and the discriminative reaction task (DRT). (B) N1 component latency averaged across the SRT and DRT. (C) P2 component latency in the SRT (the P2 component was not detected in the DRT).

Regarding the P3 components, on the DRT there was a large delay (60 and $100 \mathrm{msec}$, for the go and no-go conditions, respectively), which was associated with amplitude reductions for both disabled groups. By contrast, on the SRT, P3 amplitude and latency were comparable in the three groups. Thus, the slowing down and reduced amplitude was selective for cognitive processing related to tasks requiring visual discrimination and different responses.

At the P3 level, we did not measure differences between the open- and closed-skill groups. This may be due to the multiple cortical sources of P3 involving a large number of cortical connections, which is associated with a wide variety of cognitive tasks (Linden, 2005). Considering that the P3 peak latency was about $20-100$ msec longer than the average reaction time, the P3 may also reflect additional processing following action execution, such as response evaluation; these processes would not be affected by different types of sports experiences.

We can speculate that the general slowing observed at the electrophysiological and behavioral level in both disabled groups may represent long-term consequences of undetected (or neglected) concussions suffered during the accident that caused the loss of lower limb function. In fact, only one disabled athlete had a diagnosis of TBI, but many of them may have sustained a hidden mild TBI. Some indications in the literature (e.g., ERP study of the oddball task, a paradigm sharing some features with the go/no-go task) support this view. In particular, Gosselin and associates (2006) reported amplitude reductions of N1, P2, and P3 components following concussions. In other studies, the effects of concussion (or mild brain trauma) were detected only for the late ERP components (P3), mostly affecting amplitude (De Beaumont et al., 2007), but also affecting latency (De Beaumont et al., 2009; Lachapelle et al., 2008). In fact, the effects of concussion on P3 were long-lasting, and could be detected 30 years after the inciting event (De Beaumont et al., 2009). In a previous study (Di Russo and Spinelli, 2009), we also observed a substantial delay in the P3 component in professional boxers; the latency of the individual subjects correlated with the amount of their

FIG. 3. Spatial distribution of the event-related potential (ERP) components studied. (A) Scalp topography of the early P1, N1, and P2 components, arising from cortical visual areas in the simple reaction task (SRT). (B) Scalp topography of the late cognitive components N2 and P3, arising from the parietal and frontal areas in the SRT and the discriminative reaction task (DRT). The N2 component was absent in the SRT. 

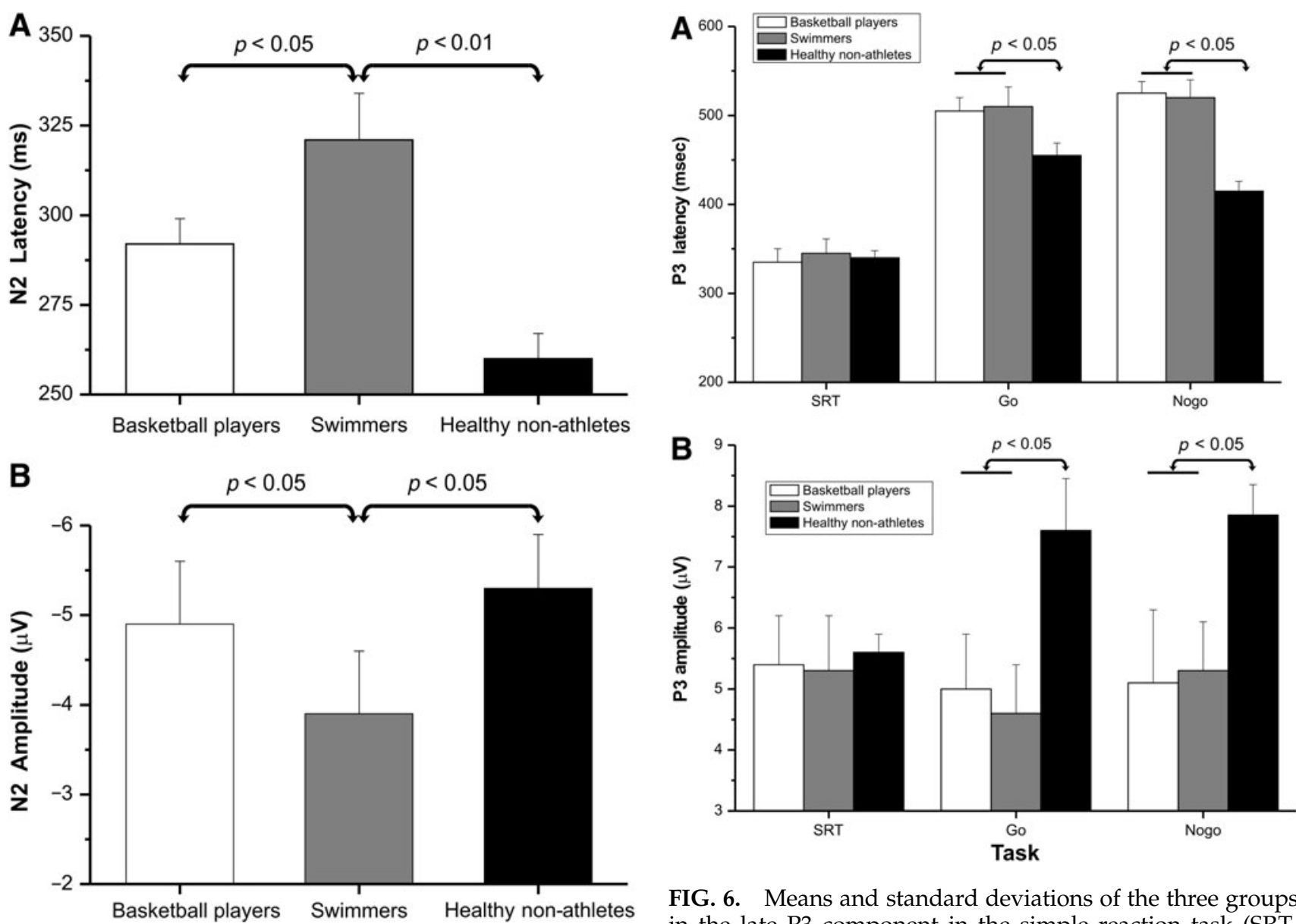

FIG. 6. Means and standard deviations of the three groups in the late P3 component in the simple reaction task (SRT, left), and the discriminative reaction task (DRT, middle and right graphs). (A) P3 latency. (B) P3 amplitude. A separate analysis of variance confirmed the absence of significant group differences in the SRT condition.

for the late N2 component. (A) Latency in go and no-go trials pooled together. (B) Amplitude in go and no-go trials pooled together.

boxing activity, and also correlated with an increasing number of blows to the head. Thus, at least some of the electrophysiological results reported in the present study, particularly the effects on the P3 component, are compatible with the idea of a long-term effect of concussion. This explanation might also apply to previous data, showing that P3 latencies are longer in amputees (Karl et al., 2004). In fact, upper-limb amputation was the result of accidents in about $80 \%$ of reported cases. Thus the long-term effects of concussion on cortical activity (on both early and late components), are relevant, and are often unexpected in the absence of a diagnosis of brain trauma.

Not all of the observed effects were due to premorbid concussion. In fact, two disabled subjects in the present study suffered from poliomyelitis (not SCI or amputation), but their data conformed to the general trend, suggesting the role of different mechanisms. Several such mechanisms have been proposed in the literature. The lack of sensory afferent impulses from the lower limbs likely plays a role (Crossman, 1996). Slowing of conduction in the cerebrospinal pathway has been invoked to explain the results of a transcranial magnetic stimulation study in an SCI group (Brouwer et al, 1992). Reduced functioning of the thalamo-cortical network was proposed to explain the differences seen in EEG between

states (open versus closed eyes) in SCI subjects (Boord et al., 2008; Tran et al., 2004). Also, a general reduction of circulatory and respiratory capacity following the pathological event, and a sedentary lifestyle may also be contributory in some cases (Plinta et al., 2005). However, this was not the case in the present study, because all disabled participants were highlevel athletes. Indeed, the results may reflect the interaction of many mechanisms. Future studies will likely reveal the contributions of the different mechanisms responsible for the observed phenomena.

In the end, we must acknowledge a potential intrinsic limitation of the present study: we cannot exclude that disabled individuals who decided to play basketball might have had better executive functioning prior to training than their peers who chose swimming. With this caveat (which could be resolved only by longitudinal experiments in which disabled individuals are randomly assigned to different sports), we propose that different sports activities might have different effects in disabled individuals. Participating in an open-skill sport such as basketball stimulates specific types of executive functions. Compared to swimmers, on the behavioral task, which involved visual stimulus recognition, stimulus response mapping, and motor response/inhibition, the basketball players showed less variability in reaction times, fewer 
costs in switching from action inhibition to action execution, and higher control of action inhibition, than swimmers. At the cortical level, as indicated by the N2 component, action control was faster and stronger in the wheelchair basketball players. These athletes were comparable to healthy non-athletes in all behavioral and cortical aspects. Thus the practice of open-skill sports may facilitate recovery of executive functions in physically-disabled patients.

\section{Author Disclosure Statement}

No competing financial interests exist.

\section{References}

Ament, P.A., Cohen, M.J., Schandler, S.L., Sowa, M., and Vulpe, M. (1995). Auditory P3 event related potentials (ERP) and brainstem auditory evoked responses (BAER) after spinal cord injury in humans. J. Spinal Cord Med. 18, 208-215.

Boord, P., Siddal, P.J., Tran, Y., Herbert, D., Middleton, J., and Craig, A. (2008). Electroencephalographic slowing and reduced reactivity in neuropathic pain following spinal cord injury. Spinal Cord 46, 118-123.

Brouwer, B., Bugaresti, J., and Ashby, P. (1992). Changes of corticospinal facilitation of lower limb spinal motor neurons after spinal cord lesions. J. Neurol. Neurosurg. Psychiatry 55, 20-24.

Cohen, M.J., Ament, P.A., Schandler, S.L., Vulpe, M. (1996). Changes in the P300 component of the tactile event-related potential following spinal cord injury. Paraplegia 34, 107-112.

Crossman, M.W. (1996). Sensory deprivation in spinal cord injury: an essay. Spinal Cord 34, 573-577.

Curran, T., Hills, A., Patterson, M.B., and Strauss, M.E. (2001). Effects of aging on visuospatial attention: and ERP study. Neuropsychologia 39, 288-301.

De Beaumont, L., Theoret, H., Mongeon, D., Messier, J., Leclerc, S., Tremblay, S., Ellemberg, D., and Lassonde, M. (2009). Brain function decline in healthy retired athletes who sustained their last sports concussion in early adulthood. Brain 132, 695-708.

Di Russo, F., and Spinelli, D. (2009). Sport is not always healthy: Executive brain dysfunction in professional boxers. Psychophysiology 43, 425-434.

Di Russo, F., Incoccia, C., Formisano, R., Sabatini, U., and Zoccolotti, P. (2005a). Abnormal motor preparation in severe traumatic brain injury with good recovery. J. Neurotrauma 22, 297-312.

Di Russo, F., Martínez, A., and Hillyard, S.A. (2003). Source analysis of event-related cortical activity during visuo-spatial attention. Cerebral Cortex 13, 486-499.

Di Russo, F., Pitzalis, S., Aprile, T., and Spinelli, D. (2005b). Effect of practice on brain activity: an investigation in top-level rifle shooters. Med. Sci. Sports Exerc. 37, 1586-1593.

Di Russo, F., Taddei, F., Aprile, T., and Spinelli, D. (2006). Neural correlates of fast stimulus discrimination and response selection in top-level fencers. Neuroscience Lett. 408, 113-118.

Fallgatter, A.J., and Strik, W.K. (1999). The NoGo-anteriorization as a neurophysiological standard-index for cognitive response control. Int. J. Psychophysiol. 32, 233-238.

Gioia, M.C., Cerasa, A., Di Lucente, L., Brunelli, S., Castellano, V., and Traballesi, M. (2005). Psychological impact of sports activity in spinal cord injury patients. Scand. J. Med. Sci. Sports 16, 412-416.

Gosselin, N., Theriault, M., Leclerc, S., Montplasir, J., and Lasonde, M. (2006). Neurophysiological anomalies in symp- tomatic and asymptomatic concussed athletes. Neurosurgery $58,1151-1161$.

Hatfield, B.D., and Hillman, C.H.(2001). The psychology of sport. A mechanistic understanding of the psychology of superior performance, in: Handbook of Sport Psychology, 2nd ed. R.N. Singer, H. Hausenblas, and C. Janelle (eds). John Wiley \& Sons: New York, pps. 362-388.

Karl, A., Diers, M., and Flor, H. (2004). P300-amplitudes in upper limb amputees with and without phantom limb pain in a visual oddball paradigm. Pain 110, 40-48.

Lachapelle, J., Bolduc-Teasdale, J., Ptito, A., McKerral, M. (2008). Defits in complex visual information processing after mild TBI: Electrophysiological markers and vocational outcome prognosis. Brain Injury 22, 265-274.

Linden, D.E. (2005). The P300: where in the brain is it produced and what does it tell us? Neuroscientist 11, 563-576.

Martínez, A., Teder-Sälejärvi, W., Vazquez, M., Molholm, S., Foxe, J.J., Javitt, D.C., Di Russo, F., Worden, M.S., and Hillyard, S.A. (2006). Objects are highlighted by spatial attention. J. Cognitive Neurosci. 18, 298-310.

McVeigh, S.A., Hitzig, M.A., and Craven, B.C. (2009). Influence of sport participation on community integration and quality of life: A comparison between sport participants and non-sport participants with spinal cord injury. J. Spinal Cord Med. 32, 115-124.

Murakami, T., Sakuma, K., and Nakashima, K. (2008). Somatosensory evoked potentials and high-frequency oscillations in athletes. Clin. Neurophysiol. 119, 2862-2869.

Nakata, H., Yoscie, M., Miura, A., and Kudo, K. (2010). Characteristics of the athletes' brain: Evidence from neurophysiology and neuroimaging. Brain Res. Rev. 62, 197-211.

Özmerdivenli, R., Bulut, S., Bayar, H., Karacabey, K., Ciloglu, F., Peker, I., and Tan, U. (2005). Effects of exercise on visual evoked potentials. Int. J. Neurosci. 115, 1043-1050.

Plinta, R., Saulicz, E., Gnat, R., Juras, G., and Waskiewicz, Z. (2005). Assessment of specific coordination in subjects with handicapped locomotor system. J. Human Kinetics 14, 41-50.

Pliszka, S.R., Liotti, M., and Woldorff, M.G. (2000). Inhibitory control in children with attention-deficit/hyperactivity disorder: event-related potentials identify the processing component and timing of an impaired right-frontal responseinhibition mechanism. Biol. Psychiatry 48, 238-246.

Salisbury, D.F., Griggs, C.B., Shenton, M.E., and McCarley, R.W. (2004). The NoGo P300 'anteriorization' effect and response inhibition. Clin. Neurophysiol. 115, 1550-1558.

Stuss, D.T., Murphy, K.J., Binns, M.A., and Alexander, M.P. (2003). Staying on the job: the frontal lobes control individual performance variability. Brain 126, 2363-2380.

Tran, Y., Boord, P., Middleton, J., and Craig, A. (2004). Levels of brain wave activity (8-13) in persons with spinal cord injury. Spinal Cord 42, 73-79.

Wang, Y.T., Chen, S., Limroongreungrat, W., and Change, L.S. (2005). Contributions of selected fundamental factors to wheelchair basketball performance. Med. Sci. Sports Exerc. 37, 130-137.

Address correspondence to: Francesco Di Russo, Ph.D. University of Rome "Foro Italico" Piazza Lauro De Bosis, 15 00135 Rome, Italy

E-mail: francesco.dirusso@uniroma4.it 
